\title{
Morphological stability, cellular viability and stem cell marker expression of three-dimensional cultures of stem cells from bone marrow and periodontium
}

\author{
JAE-YONG TAE* , HYUNJIN LEE* , HYUNA LEE, YOUNGMIN SONG and JUN-BEOM PARK
}

\author{
Department of Periodontics, College of Medicine, The Catholic University of Korea, Seoul 06591, Republic of Korea
}

Received March 24, 2020; Accepted October 19, 2020

DOI: $10.3892 /$ br_br.2020.1385

\begin{abstract}
The aim of the present study was to evaluate the morphology, cellular viability and stem cell marker expression of three-dimensional cultures of bone marrow and gingiva-derived stem cells in different ratios. Stem cell spheroids were made with bone marrow and gingiva-derived stem cells using ratios of 6:0 (Group 1), 4:2 (Group 2), 3:3 (Group 3), 2:4 (Group 4) and 0:6 (Group 5), respectively. The viability of cell spheroids was analyzed using a Live/Dead kit assay and a Cell Counting Kit-8 assay. Total RNA extraction and reverse transcription-quantitative PCR were performed to detect the mRNA expression levels of Nanog and $\beta$-actin in each group. Stem cell spheroids were well formed in silicone elastomer-based concave microwells with different ratios of bone marrow and gingiva-derived stem cells. The shape of the spheroids and their viability were maintained throughout the entirety of the experimental procedure. Statistically significant increases in spheroid diameters were noted in Groups 4 and 5 on day 1 when compared with Group 1 on day 1 . There was a significant increase in the cell viability values seen in Group 3 on day 1 when compared with Group 1 on day 1. Highest levels of Nanog expression was seen in Group 3 on day 10 , but the increase was not significant when compared with Group 1 on day 1. Co-culturing with higher ratios of gingiva-derived stem cells produced stem cell spheroids with larger diameters and increased cellular viability. This co-culture technique may be used in stem cell therapy with allogenic stem cell transplantation.
\end{abstract}

\section{Introduction}

Pluripotent stem cells have the ability to self-organize into three-dimensional aggregates termed cell spheroids or

Correspondence to: Dr Jun-Beom Park, Department of Periodontics, College of Medicine, The Catholic University of Korea, 222 Banpo-daero, Seocho-gu, Seoul 06591, Republic of Korea E-mail: jbassoonis@yahoo.co.kr

*Contributed equally

Key words: bone marrow, cellular spheroids, gingiva, organ culture techniques, stem cells organoids (1). The three-dimensional cultures, such as stem cells spheroids are shown to maintain cell survival and cell function (2). In a previous study, three-dimensional cultures preserved the cell viability and three-dimensional morphology, whilst exhibiting increased cellular function when compared with two-dimensional monolayer culture (3). These multicellular spheroids from induced pluripotent stem cells and three-dimensional culture platforms have been used for testing cell-cell interactions, drug sensitivity, anticancer activity, immune activation and organoid generation $(4,5)$.

Spheroid cultures are gaining increasing interest, particularly in the field of tissue regeneration (6). Spheroid cultures exhibit increased secretion of cytokines, including granulocyte colony stimulating factor and vascular endothelial growth factor when compared with two-dimensional cultures (7). Various methods including spinner flasks, hanging drops, non-adhesive surfaces, and microwells have been used for spheroid production (5). Amongst these methods, microwells with a three-dimensional concave geometry can produce uniformly sized stem cell spheroids with reproducible results (8). Various types of cells from different anatomical regions have been used for three-dimensional cultures (9-11). Spheroids created from a co-culture of different cell types of stem cells and endothelial cells exhibit enhanced functionality (12). Similarly, the use of multicellular spheroids composed of two types of cells results in increased osteogenic potential (13). In light of the promising findings of previous studies on co-culture techniques, the aim of the present study was to evaluate the morphology, cellular viability and expression of stem cell markers of three-dimensional cultures established using bone marrow and/or gingiva-derived stem cells in different ratios.

\section{Materials and methods}

Fabrication of cell spheroids using human bone marrow and/or human gingiva-derived stem cells. The Institutional Review Board examined and approved the present study (grant no. KC20SISE0703). Stem cell spheroids were fabricated in silicone elastomer-based microwells that were concave in shape with a $600 \mu \mathrm{m}$ diameter (cat. no. H389600; StemFIT $3 \mathrm{D}$; MicroFIT). A total of $1 \times 10^{6}$ gingiva-derived stem cells and/or bone marrow-derived stem cells were seeded in per well. Gingiva-derived stem cells were obtained as described 
previously (14). Human bone marrow-derived mesenchymal stem cells (Catholic MASTER Cells) were obtained from the Catholic Institute of Cell Therapy (15), and informed consent was obtained from all participants. The experiments were performed in accordance with the relevant guidelines and regulations specified in the Declaration of Helsinki (16). The ratios between human bone marrow-derived mesenchymal stem cells and gingiva-derived stem cells were: 6:0, Group 1; 4:2, Group 2; 3:3, Group 3; 2:4, Group 4; and 0:6, Group 5 (13). Cell aggregation and cell-spheroid formation were observed using an inverted microscope.

Determination of cell viability. The viability of spheroids was qualitatively analyzed using a Live/Dead kit assay (Molecular Probes) on day 1 (17). Stem cell spheroids were cultured in $\alpha$-minimal essential medium ( $\alpha$-MEM; Gibco; Thermo Fisher Scientific, Inc.) containing 15\% FBS (Gibco; Thermo Fisher Scientific, Inc.), $100 \mathrm{U} / \mathrm{ml}$ penicillin, $100 \mu \mathrm{g} / \mathrm{ml}$ streptomycin (Sigma-Aldrich; Merck KGaA), 200 mM L-Glutamine (Sigma-Aldrich; Merck KGaA) and $10 \mathrm{mM}$ ascorbic acid 2-phosphate (Sigma-Aldrich; Merck KGaA). These spheroids were washed twice with growth media. A suspension containing calcein acetoxymethyl ester working solution ethidium homodimer-1 was added and incubated at room temperature for $30 \mathrm{~min}$. The spheroids were observed under a fluorescence microscope on days 3 and 5 (magnification, x100).

Qualitative cellular viability analysis was performed on days 1, 3, 5 and 7 using a Cell Counting Kit-8 (CCK-8) assay (Dojindo Molecular Technologies, Inc.) (18). The spheroids were incubated for $45 \mathrm{~min}$ at $37^{\circ} \mathrm{C}$ and the spectrophotometric absorbance was measured at $450 \mathrm{~nm}$.

Reverse transcription-quantitative $(R T-q) P C R$. Cells were harvested on days 7 and 10. The total RNA was isolated using a GeneJET RNA Purification kit according to the manufacturer's protocol (Thermo Fisher Scientific, Inc.); 1 ng total RNA was used as a template for reverse transcription using SuperScript II Reverse Transcriptase (Invitrogen; Thermo Fisher Scientific, Inc.), and quantities were determined by spectrophotometry on a NanoDrop 2000 (Thermo Fisher Scientific, Inc.) at 260 and $280 \mathrm{~nm}$ on days 7 and 10.

mRNA expression was detected using qPCR with a SYBR-Green Real-Time PCR MasterMix (Enzynomics) according to the manufacturer's protocol (19). The sense and antisense primers were designed based on GenBank. The primer sequences were as follows: Nanog (accession no. NM_001297698.2) forward,5'-AGTCCCAAAGGCAAACAA CCCACTTC-3' and reverse, 5'-TGCTGGAGGCTGAGGTAT TTCTGTCTC-3'; and $\beta$-actin (accession no. NM_001101.5) forward, 5'-TGGCACCCAGCACAATGAA-3' and reverse, 5'-CTAAGTCATAGTCCGCCTAGAAGCA-3'; the mRNA levels were normalized to $\beta$-actin and expressed as the fold change (20). The mRNA expression was detected by qPCR using SYBR Green Real-Time PCR MasterMix (Enzynomics, Daejeon, South Korea) according to the manufacturer's protocol.

Statistical analysis. Statistical analysis was performed using SPSS version 12 (SPSS, Inc.) Data are presented as the mean \pm standard deviation. A test of normality was performed, and a one-way ANOVA with a post-hoc Tukey's test, or a Kruskal Wallis test with Bonferroni corrected Mann-Whitney-U test was performed to determine differences between the groups. $\mathrm{P}<0.05$ was considered to indicate a statistically significant difference.

\section{Results}

Evaluation of cell morphology. Stem cell spheroids were well formed in the silicone elastomer-based concave microwells with only bone marrow-derived stem cells on days 1, 3, 5 and 7 (Fig. 1A). There were no significant changes in the morphology with the different ratios of bone marrow and gingiva-derived stem cells on days 1 and 3 . In general, the shapes of the cells on day 5 were similar to the shapes in each group on day 1 . There were no significant changes in the morphology with the longer incubation time of 7 days.

The average spheroid diameter in each group on days 1, 3, 5 and 7 are shown in Fig. 1B. The average spheroid diameter for Groups 1, 2, 3, 4 and 5 on day 1 were 152.7 $\pm 2.1,187.4 \pm 27.6$, $168.9 \pm 7.4,207.1 \pm 10.2$ and $224.9 \pm 5.1 \mu \mathrm{m}$, respectively $(\mathrm{P}<0.05)$. There were statistically significant increases in Groups 4 and 5 on day 1 when compared with Group 1 on day 1 . The average spheroid diameters for Groups 1, 2, 3, 4 and 5 on day 3 were $151.6 \pm 9.3,169.9 \pm 7.7,152.5 \pm 4.9,179.7 \pm 10.9$ and $185.1 \pm 12.9 \mu \mathrm{m}$, respectively. The diameters of the spheroids appeared to increase the ratio of gingiva-derived stem cells increased.

Determination of cell viability. The cellular viability was determined using a Live/Dead kit assay using a fluorescent microscope and is shown in Fig. 2. Most of the cells in the spheroids emitted green fluorescence, and the morphology was round without significant changes at day 1 .

The results of cell viability on days $1,3,5$ and 7 are shown in Fig. 3. The relative viability value of Groups 1,2,3, 4 and 5 on day 1 were $100.0 \pm 10.7 \%, 115.5 \pm 3.3 \%, 119.1 \pm 7.5 \%, 115.4 \pm 3.4 \%$ and $116.8 \pm 5.4 \%$, respectively $(\mathrm{P}<0.05)$. There were significant increases in the values seen in Groups 3 when compared with Group 1 on day 1.

Validation of $m R N A$ expression using RT-qPCR. RT-qPCR was performed to assess the mRNA expression levels of Nanog and $\beta$-actin on days 7 and 10 (Fig. 4). The mRNA levels were normalized to $\beta$-actin levels and expressed as a fold change. The relative expression of Nanog in Groups 1,2, 3, 4 and 5 on day 7 were $100.0 \pm 8.3 \%, 77.6 \pm 22.1 \%, 98.6 \pm 19.6 \%, 65.7 \pm 33.9 \%$ and $64.8 \pm 32.7 \%$, respectively (Fig. 4A). The relative expression of Nanog in Groups 1, 2, 3, 4 and 5 on day 10 were $100.0 \pm 1.2$, $102.1 \pm 16.0,126.7 \pm 12.0,101.2 \pm 14.2$ and $124.1 \pm 21.3 \%$, respectively, with the highest value observed in Group 3 (Fig. 4B). However, no significant differences in Nanog expression were observed in Groups 2-5 when compared with Group 1 ( $\mathrm{P}>0.05$; Fig. 4A and B).

\section{Discussion}

In the present study, stem-cell spheroids were formed with human bone marrow and gingiva-derived stem cells using concave microwells. This study clearly showed that the shape of the spheroids were maintained throughout the entirety of 

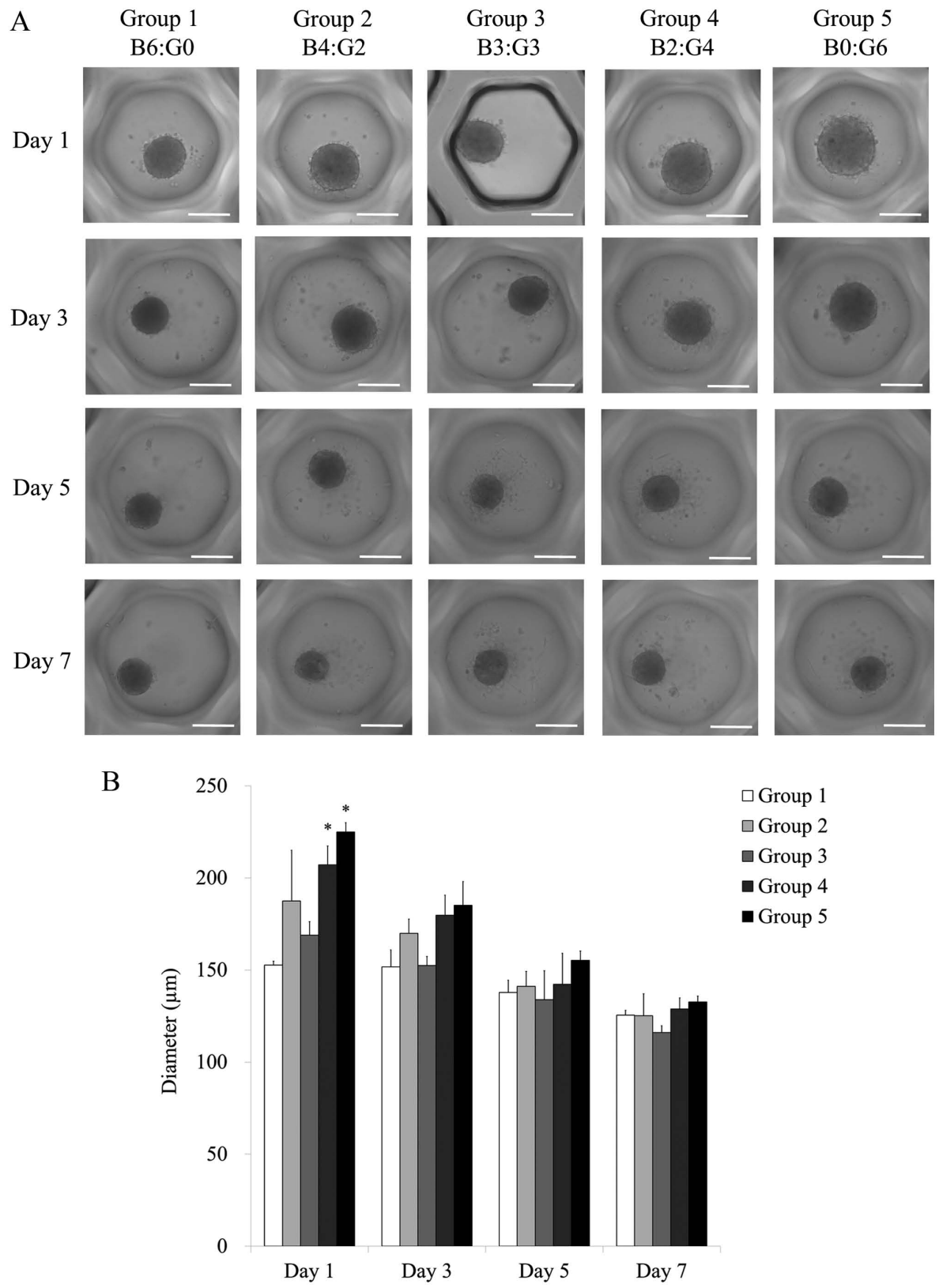

$\square$ Group 1
$\square$ Group 2
— Group 3
- Group 4
- Group 5

Day 3

Day 5

Day 7

Figure 1. Morphology and diameter of co-culture of bone marrow and gingiva-derived stem cells. (A) Morphology of the cell spheroids on days 1,3, 5 and 7. Magnification, x200. Scale bar, $200 \mu \mathrm{m}$. (B) Diameter of cell spheroids on days 1,3, 5 and 7. ${ }^{*} \mathrm{P}<0.05$ vs. Group 1 on day 1.

the experimental procedure and increased cellular viability was achieved when using a co-culture of bone marrow and gingiva-derived stem cells.

A co-culture method has been used in previous reports to generate spheroids $(21,22)$. The co-culture system of endothelial cells and adipose-derived stem cells led to the promotion of vascular morphogenesis in microvessel structures (21). Higher cell-cell interactions were noted when bone marrow-derived stem cells were co-cultured with glioblastoma multiform cell lines (22). In the present study, stem cells derived from different anatomical regions including bone marrow and gingival were used for analysis, and the results showed that this approach can be useful when there is a limited number of cells of each type.

Stem cells derived from different anatomical regions have been used for various applications. Spheroids fabricated with several types of cells, including bone marrow-derived stem cells for Bio 3D printer-produced histological chondrogenesis and vasculogenesis (10). Similarly, adipose-derived stem cells can be used as a promising therapeutic approach for soft tissue healing (11). A previous study showed that human periodontal ligament-derived stem cells enhanced retinal ganglion cell survival, and this approach can be used for protection against optic neuropathy (9). Gingiva-derived stem cells have various advantages, as the cells can be obtained under local anesthesia without severe complications $(23,24)$. Applying $\geq 2$ types of cells may complement the limitations in each type.

RT-qPCR was performed to detect the mRNA expression levels of Nanog and $\beta$-actin in each group. Nanog is a transcription factor that is involved in self-renewal of undifferentiated stem cells (25). Nanog has been shown to be expressed in several types of tumors, highlighting the potential presence of 


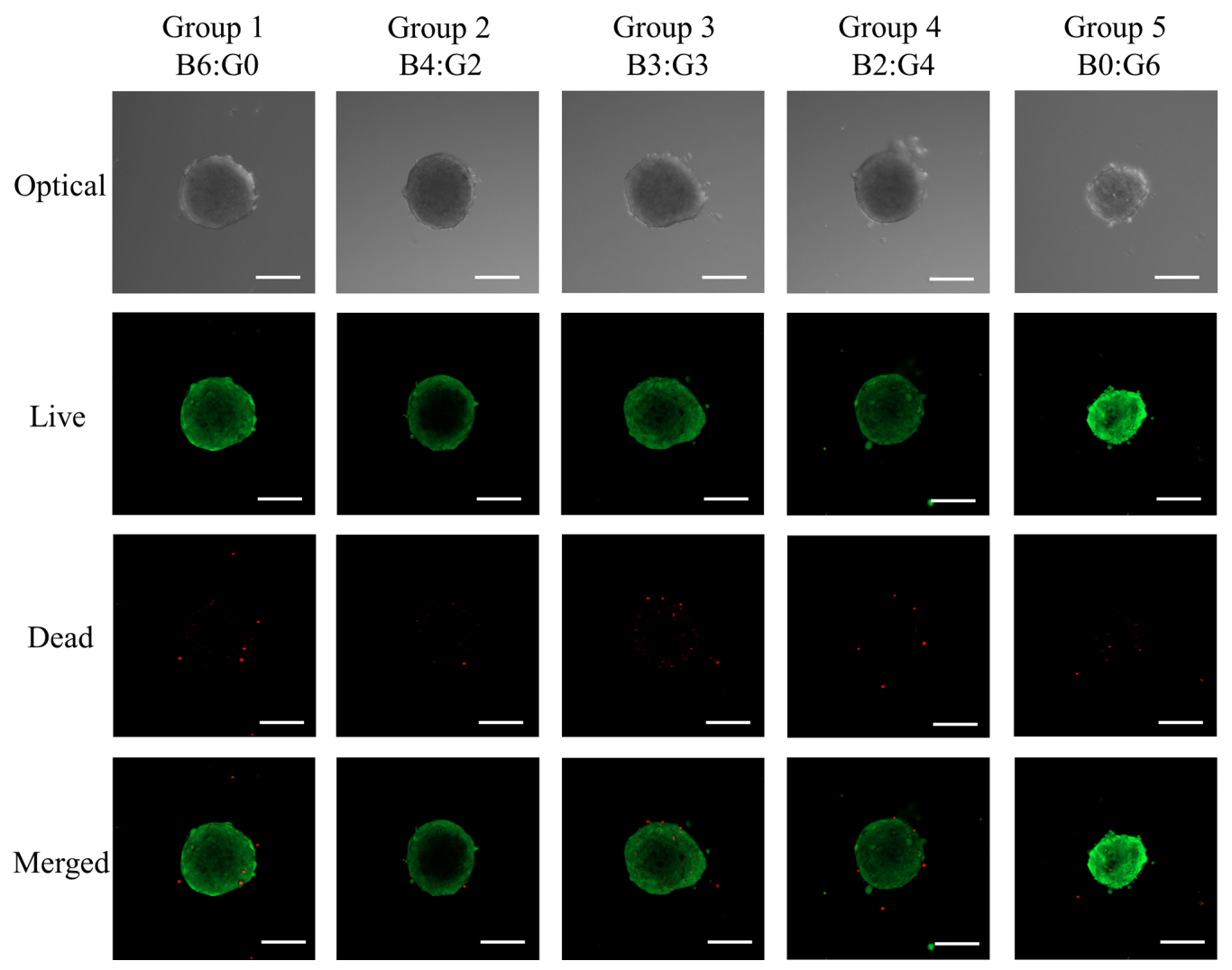

Figure 2. Qualitative results of cellular viability observed using a confocal microscope on day 1. Optical, live and dead images are provided. Magnification x100. Scale bar, $200 \mu \mathrm{m}$. B, bone marrow-derived stems cells; G, gingiva-derived stem cells.

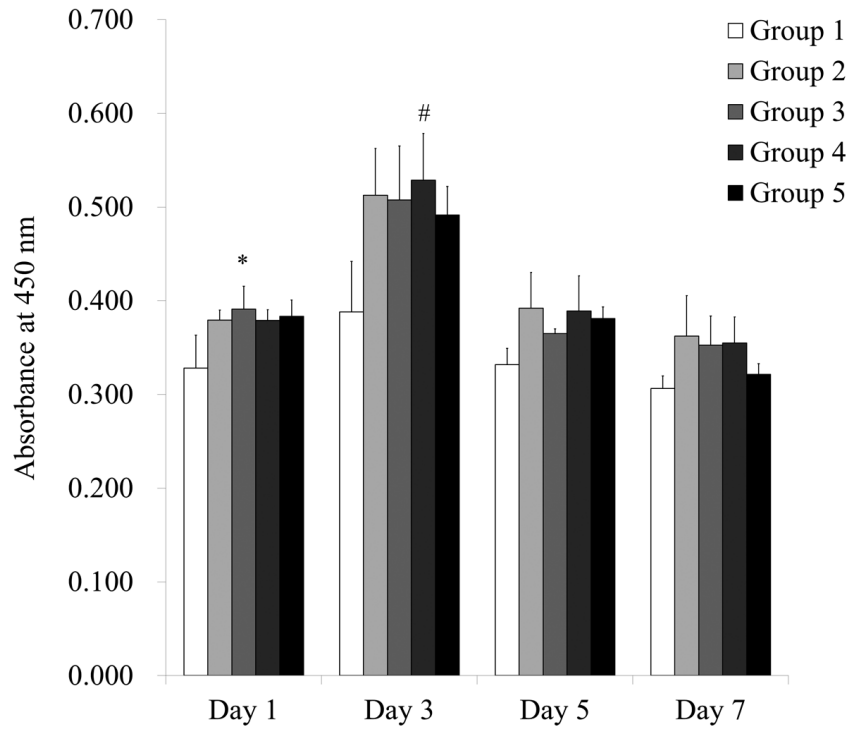

Figure 3. Cellular viability of the stem cells on days $1,3,5$ and 7 using a Cell Counting Kit-8 assay. ${ }^{*} \mathrm{P}<0.05$ vs. Group 1 on day $1 .{ }^{*} \mathrm{P}<0.05$ vs. with Group 1 on day 3 .

a population of cancer stem cells (26). In the present study, no significant changes in Nanog expression were observed between the groups with different ratios of cells.

There are several limitations in the present study that should be considered when interpretating the results. Nanog expression has been used as a stem cell marker. However, there are various markers for evaluating the characteristics of stem

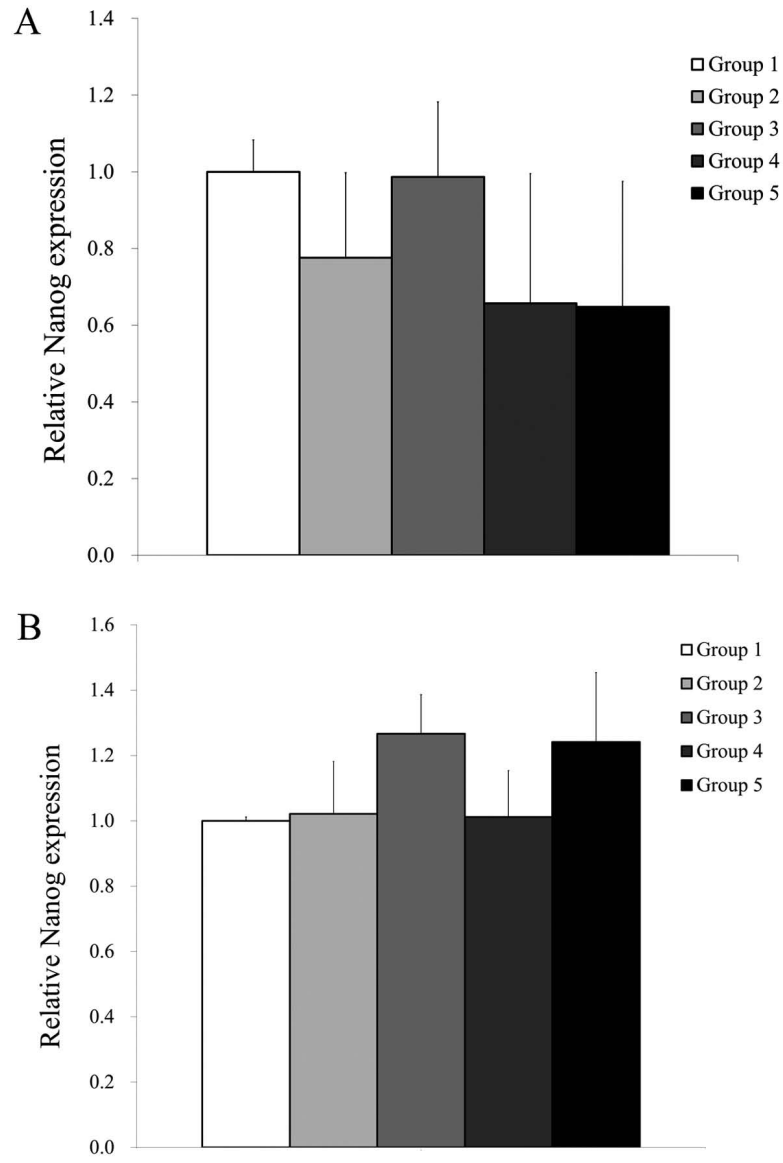

Figure 4. Reverse transcription-quantitative PCR of Nanog expression. Expression of Nanog on days (A) 7 and (B) 10. 
cells and CD73 and CD90 have been widely served as positive markers of stem cells (27). Cellular viability was evaluated qualitatively using a Live/Dead kit and quantitatively using a CCK-8 assay. Caspase activity can be used to evaluate the apoptosis of stem cells (28). The differential behaviors between the groups can be explained based on cell-cell interactions. These cell-cell interactions can be visualized using photo excitation of fluorescent proteins (29). Evaluation of additional adipogenic and chondrogenic differentiation potential may broaden the applicability for various purposes.

In conclusion, the present study demonstrated that stem cell spheroids could be formed with human bone marrow and gingiva-derived stem cells using concave microwells. The shape of the spheroids were maintained throughout the entirety of the experimental procedure. The use of co-cultures with higher ratios of gingiva-derived stem cells produced stem cell spheroids with larger diameters. Highest cellular viability and highest levels of Nanog expression was achieved with co-culture of bone marrow and gingiva-derived stem cells. This co-culture technique may be used for stem cell therapy with allogenic stem cell transplantation. Further studies regarding cell-cell interactions should be performed to analyze the underlying mechanisms. Application of stem cell spheroids formed of bone marrow and gingiva-derived stem cells using various ratios in in vivo models are warranted to evaluate their therapeutic efficacy.

\section{Acknowledgements}

Not applicable.

\section{Funding}

This study was supported by the National Research Foundation of Korea grant funded by the Korea government (MSIT) (grant no. 2020R1A2C4001624), and supported by research funding from Seoul St. Mary's Hospital, The Catholic University of Korea.

\section{Availability of data and materials}

All data generated or analyzed during this study are included in the published article.

\section{Authors' contributions}

J-YT, HyunjinL, HyunaL, YS and J-BP designed the study, analyzed the data, performed the experiments as well as wrote and reviewed the manuscript. All authors have read and approved the final manuscript.

\section{Ethics approval and consent to participate}

The present study was reviewed and approved by the Institutional Review Board of Seoul St. Mary's Hospital, College of Medicine, the Catholic University of Korea (approval no. KC20SISE0703). Informed consent was obtained from all participants. All experiments were performed in accordance with relevant guidelines and regulations specified in the Declaration of Helsinki.

\section{Patient consent for publication}

Not applicable.

\section{Competing interests}

The authors declare that they have no competing interests.

\section{References}

1. Pasca SP: The rise of three-dimensional human brain cultures. Nature 553: 437-445, 2018.

2. Mazza G, Al-Akkad W, Rombouts K and Pinzani M: Liver tissue engineering: From implantable tissue to whole organ engineering. Hepatol Commun 2: 131-141, 2017.

3. Ong SM, Zhang C, Toh YC, Kim SH, Foo HL, Tan $\mathrm{CH}$, van Noort D, Park S and Yu H: A gel-free 3D microfluidic cell culture system. Biomaterials 29: 3237-3244, 2008.

4. Pamies D, Block K, Lau P, Gribaldo L, Pardo CA, Barreras P, Smirnova L, Wiersma D, Zhao L, Harris G, et al: Rotenone exerts developmental neurotoxicity in a human brain spheroid model. Toxicol Appl Pharmacol 354: 101-114, 2018.

5. Lee GH, Suh Y and Park JY: A paired bead and magnet array for molding microwells with variable concave geometries. J Vis Exp 28: 55548, 2018

6. Bauman E, Feijao T, Carvalho DTO, Granja PL and Barrias CC: Xeno-free pre-vascularized spheroids for therapeutic applications. Sci Rep 8: 230, 2018.

7. Redondo-Castro E, Cunningham CJ, Miller J, Brown H, Allan SM and Pinteaux E: Changes in the secretome of tri-dimensional spheroid-cultured human mesenchymal stem cells in vitro by interleukin-1 priming. Stem Cell Res Ther 9: 11, 2018.

8. Lee SI, Yeo SI, Kim BB, Ko Y and Park JB: Formation of size-controllable spheroids using gingiva-derived stem cells and concave microwells: Morphology and viability tests. Biomed Rep 4: 97-101, 2016.

9. Cen LP, Ng TK, Liang JJ, Zhuang X, Yao X, Yam GHF, Chen H, Cheung HS, Zhang M and Pang CP: Human periodontal ligament-derived stem cells promote retinal ganglion cell survival and axon regeneration after optic nerve injury. Stem Cells 36: 844-856, 2018

10. Taniguchi D, Matsumoto K, Tsuchiya T, Machino R, Takeoka Y, Elgalad A, Gunge K, Takagi K, Taura Y, Hatachi G, et al: Scaffold-free trachea regeneration by tissue engineering with bio-3D printing. Interact Cardiovasc Thorac Surg 26: 745-752, 2018.

11. Oberringer M, Bubel M, Jennewein M, Guthörl S, Morsch T, Bachmann S, Metzger $\mathrm{W}$ and Pohlemann T: The role of adipose-derived stem cells in a self-organizing 3D model with regard to human soft tissue healing. Mol Cell Biochem 445: 195-210, 2018.

12. Tae JY, Lee SI, Ko Y and Park JB: Enhanced osteogenic differentiation potential of stem-cell spheroids created from a coculture of stem cells and endothelial cells. Implant Dent 26: 922-928, 2017.

13. Tae JY, Lee H, Lee H, Ko Y and Park JB: Osteogenic potential of cell spheroids composed of varying ratios of gingiva-derived and bone marrow stem cells using concave microwells. Exp Ther Med 16: 2287-2294, 2018.

14. Jin SH, Lee JE, Yun JH, Kim I, Ko Y and Park JB: Isolation and characterization of human mesenchymal stem cells from gingival connective tissue. J Periodontal Res 50: 461-467, 2015.

15. Jeong CH, Kim SM, Lim JY, Ryu CH, Jun JA and Jeun SS: Mesenchymal stem cells expressing brain-derived neurotrophic factor enhance endogenous neurogenesis in an ischemic stroke model. Biomed Res Int 2014: 129145, 2014.

16. World Medical Association: World medical association declaration of helsinki: Ethical principles for medical research involving human subjects. JAMA 310: 2191-2194, 2013.

17. Kang SH, Park JB, Kim I, Lee W and Kim H: Assessment of stem cell viability in the initial healing period in rabbits with a cranial bone defect according to the type and form of scaffold. J Periodontal Implant Sci 49: 258-267, 2019.

18. Tae JY, Ko Y and Park JB: Evaluation of fibroblast growth factor-2 on the proliferation of osteogenic potential and protein expression of stem cell spheroids composed of stem cells derived from bone marrow. Exp Ther Med 18: 326-331, 2019. 
19. Lee H, Min SK, Song Y, Park YH and Park JB: Bone morphogenetic protein-7 upregulates genes associated with osteoblast differentiation, including collagen I, Sp7 and IBSP in gingiva-derived stem cells. Exp Ther Med 18: 2867-2876, 2019.

20. Livak KJ and Schmittgen TD: Analysis of relative gene expression data using real-time quantitative PCR and the 2(-Delta Delta $\mathrm{C}(\mathrm{T})$ ) method. Methods 25: 402-408, 2001.

21. Kook YM, Kim H, Kim S, Heo CY, Park MH, Lee K and Koh WG: Promotion of vascular morphogenesis of endothelia cells co-cultured with human adipose-derived mesenchymal stem cells using polycaprolactone/gelatin nanofibrous scaffolds. Nanomaterials (Basel) 8: 117, 2018.

22. Oliveira MN, Pillat MM, Motaln H, Ulrich H and Lah TT: Kinin-B1 receptor stimulation promotes invasion and is involved in cell-cell interaction of co-cultured glioblastoma and mesenchymal stem cells. Sci Rep 8: 1299, 2018.

23. Lee SI, Ko Y and Park JB: Evaluation of the maintenance of stemness, viability, and differentiation potential of gingiva-derived stem-cell spheroids. Exp Ther Med 13: 1757-1764, 2017.

24. Ha DH, Pathak S, Yong CS, Kim JO, Jeong JH and Park JB Potential differentiation ability of gingiva originated human mesenchymal stem cell in the presence of tacrolimus. Sci Rep 6: 34910, 2016.
25. Takahashi K, Tanabe K, Ohnuki M, Narita M, Ichisaka T, Tomoda $\mathrm{K}$ and Yamanaka S: Induction of pluripotent stem cells from adult human fibroblasts by defined factors. Cell 131: 861-872, 2007.

26. Zhou X, Zhou YP, Huang GR, Gong BL, Yang B, Zhang DX, $\mathrm{Hu} \mathrm{P}$ and $\mathrm{Xu} \mathrm{SR}$ : Expression of the stem cell marker, Nanog, in human endometrial adenocarcinoma. Int J Gynecol Pathol 30: 262-270, 2011.

27. Lee H, Min SK and Park JB: Effects of demographic factors on adipogenic and chondrogenic differentiation in bone marrow-derived stem cells. Exp Ther Med 17: 3548-3554, 2019.

28. Khoshlahni N, Sagha M, Mirzapour T, Zarif MN and Mohammadzadeh-Vardin M: Iron depletion with deferoxamine protects bone marrow-derived mesenchymal stem cells against oxidative stress-induced apoptosis. Cell Stress Chaperones 25: 1059-1069, 2020.

29. Dustin ML: Visualization of cell-cell interaction contacts: Synapses and kinapses. Self Nonself 2: 85-97, 2011.

(1)(3) This work is licensed under a Creative Commons Attribution-NonCommercial-NoDerivatives 4.0 International (CC BY-NC-ND 4.0) License. 